\title{
Polymorphic expression of CYP2C19 and CYP2D6 in the developing and adult human brain causing variability in cognition, risk for depression and suicide: the search for the endogenous substrates
}

\author{
"Although these polymorphisms are not causing an apparent \\ phenotype, accumulating evidence indicates that they can influence \\ brain function."
}

Keywords: depression $\bullet$ endocannabinoids $\bullet$ GABA receptors $\bullet$ gene duplication $\bullet$ serotonin - ultrarapid metabolizer

\section{Introduction}

The two major cytochrome P450 polymorphisms, with respect to variable drug response, are those of CYP2C19 and CYP2D6. Both gene variations can cause abolished, reduced, normal or increased enzyme expression translating into the poor (PM), intermediate (IM), extensive (EM) and ultrarapid (UM) metabolizer phenotypes [1]. Multiple gene copies of CYP2D6 emerged in Ethiopia and North Africa in order to make the inactivation of the toxic plant alkaloids this enzyme metabolizes more efficient [2]; evaluation of the Neanderthal sequences reveals defect CYP genes [3]; these data indicate a long history of polymorphism in these genes. Although these polymorphisms are not causing an apparent phenotype, accumulating evidence indicates that they can influence brain function.

\section{CYP2D6 in the brain}

CYP2D6 has been found in neurons in numerous human brain areas, including the thalamus, hypothalamus, hippocampus, substantia nigra, cerebellum, and in several layers of the frontal neocortex $[4,5]$ raising questions about its potential role in these neurons. The enzyme was implicated in metabolism of the endogenous compounds 5-methoxytryptamine, anandamide, progesterone and tyramine and in generation of serotonin and dopamine from trace amines [6].
This hypothesis is strengthened by the fact that in a transgenic mouse model, with CYP2D6 expressed in the brain, higher serotonin levels have been measured in several brain regions, including the cerebellum and hippocampus [7]. CYP2D6 might also affect the endocannabinoid system within the CNS due to its ability to metabolize anandamide and its derivatives [8]; however, the physiological significance of such metabolism is still unclear.

\section{CYP2D6 \& brain phenotypes}

Associations between CYP2D6 genotypes and personality traits gave the first indications that CYP2D6 might have endogenous functions apart from its important role in drug metabolism. In one of the first reports, it was shown that PMs displayed higher impulsivity-related traits [9], which was confirmed by a later study [10]. Others have found PMs to be more anxiety-prone and less successful in socialization when compared with EMs [11,12]. These heterogeneous results might be related to different ethnicities of the subjects and different methods used for monitoring the personality, but do suggest that CYP2D6 might have an endogenous role in the human brain influencing behavior.

The UM CYP2D6 phenotype has been suggested to predict suicidal risk [13,14] and increased suicidal behavior among individu-

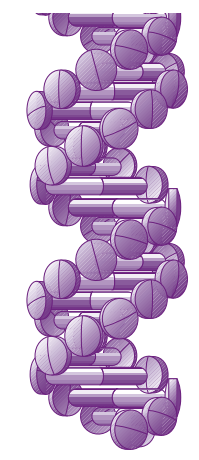

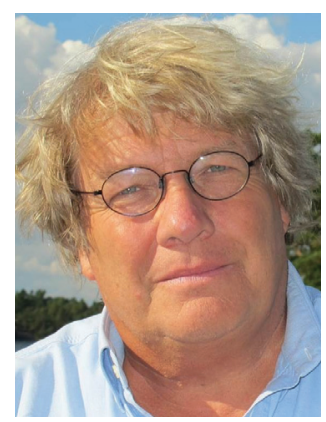

Magnus Ingelman-Sundberg Author for correspondence:

Department of Physiology

\& Pharmacology, Section of Pharmacogenetics, Karolinska Institutet, SE-17177 Stockholm, Sweden

magnus.ingelman-sundberg@ki.se

Anna Persson

Department of Physiology

\& Pharmacology, Section of Pharmacogenetics, Karolinska Institutet, SE-17177 Stockholm, Sweden

Marin M Jukic Department of Physiology \& Pharmacology, Section of Pharmacogenetics, Karolinska Institutet, SE-17177 Stockholm, Sweden

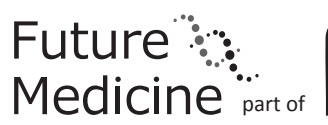
fSO 
als with eating disorders [15]. In search for a physiological effect of higher CYP2D 6 brain expression in such individuals, Stingl and collaborators examined the perfusion rates in the thalamus and the right hippocampus among healthy human subjects. They detected an effect of CYP2D6 polymorphism on resting-state perfusion in the thalamus, hypothalamus, posterior cerebral cortex, and isolated parts of the medial temporal lobe and orbitofrontal cortex, where PMs had lower perfusion rates, a pattern that suggested an association with brain circuits involved in vigilance. Also, they tested the effect of the CYP2D6 polymorphism on a standard working-memory task and recognition of facial expressions, a task eliciting neural circuits related with the detection of emotionally arousing stimuli measured with fMRI. In both studies they found an effect of the CYP2D6 polymorphism in visual areas in the posterior cerebral cortex, whereas no effect could be detected in areas outside of those staked out by the previous perfusion study (see [16] and references therein). This provides an interesting mechanistic link to the findings concerning a relationship to suicide.

\section{CYP2C19 expression in the developmental brain}

Expression of CYP2C19 in humans has long been thought to be restricted to the liver and small intestine [1]; however, using a transgenic mouse model with 12 gene copies of the CYP2C19 gene it was shown that the human CYP2C19 gene is expressed in fetal brain and silenced completely 1 week after birth [17]. Analyses of human fetal brain samples showed relatively high cortical expression levels of CYP2C19, around $0.5 \%$ of that seen in adult liver [17]. Altogether, these data indicate a transient fetal brain expression of CYP2C19 that might affect development.

Like CYP2D6, CYP2C19 has broad substrate specificity and it metabolizes many different classes of psychotropic drugs, including selective serotonin-reuptake inhibitors, tricyclic antidepressants such as amitriptyline, benzodiazepines such as diazepam, and the anticonvulsant drug mephenytoin [1].

\section{CYP2C19 \& brain phenotypes}

CYP2C19 polymorphism was associated with depressive mode in humans in study encompassing 1742 twins. Twins carrying allelic variants causing high activity of the enzyme (CYP2C19*1) showed more depressive symptoms than the subjects carrying a defective allelic variant $\left(C Y P 2 C 19^{*} 2\right)$, based on their significantly lower $\mathrm{T} 1$ scores in the center for epidemiologic studies depression (CES-D) scale and lower scores on the subscales depressed mood, psychomotor retardation, and somatic complaints, compared with extensive metabolizers [18].
The effect of the CYP2C19 genotypes on brain function and morphology was studied in the CYP2C19 transgenic mice. As adults they showed decreased hippocampal volume, an anxious phenotype, and increased hippocampal activation after acute stress [17]. The reduction in the hippocampal volume appears to be directly caused by CYP2C19 expression; the homozygotes were showing a more dramatic reduction of hippocampal volume [60\%] than hemizygotes [12\%] after birth. Reduced hippocampal volumes are commonly observed in several neuropsychiatric disorders including post-traumatic stress disorder, schizophrenia, and major depression (see [17] and references therein).

Adult CYP2C19 hemizygotes showed increased stress sensitivity and increased anxiety-like behavior. Stressful life events and stress sensitivity are major risk factors for psychiatric diseases making this animal a model of interest for factors involved in the regulation of stress response. These mice also showed a reduced number of GABAergic interneurons in the dentate gyrus of the hippocampus, the region in which $\alpha 2 \mathrm{GABA}_{\mathrm{A}}$ and not $\alpha 3 \mathrm{GABA}_{\mathrm{A}}$ receptors are present [19]. Anxiolytic effects of benzodiazepines are mediated by $\mathrm{GABA}_{\mathrm{A}}$ receptor subtypes containing $\alpha 2$ and $\alpha 3$ subunits [20]; therefore, it might be plausible that the reduced number of GABAergic interneurons in the dentate gyrus leads to hippocampus over-activation in stress and the anxious mouse phenotype by decreasing hippocampal $\alpha 2 \mathrm{GABA}_{\mathrm{A}}$ signaling.

CYP2C19 has been shown to metabolize cannabinoid compounds [21], previously shown to be important for the successful migration of GABAergic neurons to the hippocampus [22]. During hippocampal development, CB1 is present in GABA interneurons [23]; therefore, it can be hypothesized that the reduced number of GABA interneurons observed in CYP2C19 transgenic mice is caused by impaired cannabinoid signaling in developing hippocampus.

A role of CYP2C19 in the metabolism of endogenous substances during brain development seems to be a likely explanation for the phenotypes observed in the transgenic mouse model; however, the identities of these substances remain to be discovered.

\section{Synergy of increased CYP2C19 \& CYP2D6 activity}

CYP2D6 and CYP2C19 have to a great extent overlapping substrate specificities. A recent study by Llerena and collaborators examining suicide risk among subjects having both high CYP2D6 and CYP2C19 expression is interesting [24]. Thus, patients with a high CYP2D6-CYP2C19 metabolic capacity showed an increased risk for a severe suicide attempt as measured 
by the SIS-objective circumstance subscale, particularly in absence of a family history of suicide. Those with no family history of suicide are less vulnerable and consequently may need more stressful triggers to precipitate it, indicating that having a higher CYP2D6CYP2C19 metabolic capacity can be associated with the increased sensitivity to these triggers.

\section{Conclusion}

Increasing evidence suggest that the polymorphic enzymes CYP2C19 and CYP2D6 indeed have endogenous functions in the development and regulation of specific neuronal pathways, translating into interindividual differences in cognition and risk for depression and suicide. Enzyme-coding gene polymorphisms determine metabolic rates of a large number of psychotropic drugs modifying their concentrations at the site of action and can predict a need for adjustments in dosage. Since the enzymes also metabolize endo-

\section{References}

1 Ingelman-Sundberg M, Sim SC, Gomez A et al. Influence of cytochrome $\mathrm{P} 450$ polymorphisms on drug therapies: pharmacogenetic, pharmacoepigenetic and clinical aspects. Pharmacol. Ther. 3, 496-526 (2007).

2 Ingelman-Sundberg M. Genetic polymorphisms of cytochrome P450 2D6 (CYP2D6): clinical consequences, evolutionary aspects and functional diversity. Pharmacogenomics J. 1, 6-13 (2005).

3 Sankararaman S, Mallick S, Dannemann M et al. The genomic landscape of Neanderthal ancestry in present-day humans. Nature 507, 354-357 (2014).

4 Ferguson CS and Tyndale RF. Cytochrome P450 enzymes in the brain: emerging evidence of biological significance. Trends Pharmacol. Sci. 12, 708-714 (2011).

5 Dutheil F, Dauchy S, Diry M et al. Xenobiotic-metabolizing enzymes and transporters in the normal human brain: regional and cellular mapping as a basis for putative roles in cerebral function. Drug Metab. Dispos. 7, 1528-1538 (2009).

6 Miksys S, Tyndale RF. Cytochrome P450-mediated drug metabolism in the brain. J. Psychiatry Neurosci. 3, 152-163 (2013).

7 Cheng J, Zhen Y, Miksys SB et al. Potential role of CYP2D6 in the central nervous system. Xenobiotica 43, 973-984 (2013).

8 Snider NT, Sikora MJ, Sridar C et al. The endocannabinoid anandamide is a substrate for the human polymorphic cytochrome P450 2D6. J. Pharmacol. Exp. Ther. 2, 538-545 (2008).

9 Bertilsson L, Alm C, De Las Carreras C et al. Debrisoquine hydroxylation polymorphism and personality. Lancet 8637, 555 (1989).

10 Roberts RL, Luty SE, Mulder RT et al. Association between cytochrome P450 2D6 genotype and harm avoidance. Am J. Med. Genet. B Neuropsychiatr. Genet. 1, 90-93 (2004). genous psychotropic substances, it is very likely that the observed brain phenotypes involve their altered metabolism at different stages of brain development and during adulthood. Although being a difficult task, the identification of these compounds would provide an important knowledge for the molecular and cellular control of phenotypic alterations and lay a ground for possible future pharmacologic interventions.

\section{Financial \& competing interests disclosure}

The research in the authors' laboratory is supported by grants from The Swedish Research Council, The Swedish Cancer Foundation, IMI and EU-FP7. The authors have no other relevant affiliations or financial involvement with any organization or entity with a financial interest in or financial conflict with the subject matter or materials discussed in the manuscript apart from those disclosed.

No writing assistance was utilized in the production of this manuscript.

11 Llerena A, Edman G, Cobaleda J et al. Relationship between personality and debrisoquine hydroxylation capacity. Suggestion of an endogenous neuroactive substrate or product of the cytochrome P4502D6. Acta Psychiatr. Scand. 1, 23-28 (1993).

12 Gonzalez I, Penas-Lledo EM, Perez B et al. Relation between CYP2D6 phenotype and genotype and personality in healthy volunteers. Pharmacogenomics 7, 833-840 (2008).

13 Zackrisson AL, Lindblom B, and Ahlner J. High frequency of occurrence of CYP2D6 gene duplication/ multiduplication indicating ultrarapid metabolism among suicide cases. Clin. Pharmacol. Ther. 3, 354-359 (2010).

14 Stingl JC and Viviani R. CYP2D6 in the brain: impact on suicidality. Clin. Pharmacol. Ther. 3, 352-353 (2011).

15 Peñas-Lledó EM, Dorado P, Aguera Z et al. High risk of lifetime history of suicide attempts among CYP2D6 ultrarapid metabolizers with eating disorders. Mol. Psychiatry 7, 691-692 (2011).

16 Stingl JC, Brockmöller J, Viviani R. Genetic variability of drug-metabolizing enzymes: the dual impact on psychiatric therapy and regulation of brain function. Mol. Psychiatry 18, 273-287 (2013).

17 Persson A, Sim SC, Virding S et al. Decreased hippocampal volume and increased anxiety in a transgenic mouse model expressing the human CYP2C19 gene. Mol. Psychiatry 19, 733-741 (2014).

18 Sim SC, Nordin L, Andersson TM et al. Association between CYP2C19 polymorphism and depressive symptoms. Am. J. Med. Genet. B Neuropsychiatr. Genet. 153B, 1160-1166 (2010).

19 Hortnagl H, Tasan RO, Wieselthaler A et al. Patterns of mRNA and protein expression for 12 GABAA receptor subunits in the mouse brain. Neuroscience 236, 345-372 (2013). 
20 Low K, Crestani F, Keist R et al. Molecular and neuronal substrate for the selective attenuation of anxiety. Science 290 , 131-134 (2000).

21 Jiang R, Yamaori S, Takeda S, Y et al. Identification of cytochrome P450 enzymes responsible for metabolism of cannabidiol by human liver microsomes. Life Sci. 89, 165-170 (2011).

22 Berghuis P, Dobszay MB, Wang X et al. Endocannabinoids regulate interneuron migration and morphogenesis by transactivating the TrkB receptor. Proc. Natl Acad. Sci. USA 102, 19115-19120 (2005).
23 Morozov YM, Torii M, Rakic P. Origin, early commitment, migratory routes, and destination of cannabinoid type 1 receptor-containing interneurons. Cereb. Cortex 19, i78-i89 (2009).

24 Peñas-Lledó E, Guillaume S, Naranjo ME et al. A combined high CYP2D6-CYP2C19 metabolic capacity is associated with the severity of suicide attempt as measured by objective circumstances. Pharmacogenomics J. doi:10.1038/tpj.2014.42 (2014) (Epub ahead of print). 